\title{
EMBARAZO PROLONGADO
}

\section{Estado actual del problema}

\author{
Dr. Fernando Sánchez Torres*
}

Si aceptamos como "embarazo prolongado" toda gestación que va más allá de la duración normal, es necesario precisar qué se entiende por "duración normal". En todas las especies animales la duración del ciclo gravídico tiende a ser constante; en la especie humana dura aproximaclamente 280 días, a partir del primer día de la última menstruación. Este lapso corresponde a la "duración aparente", pues la "duración real" es el tiempo transcurrido desde la fecundación hasta el parto. Dado que la fertilización ocurre pocas horas después de la ovulación, es natural que la duración aparente varíe ampliamente, a causa, precisamente, de la variabilidad con que se presenta la ovulación en la mujer. Higgins (18) encontró que siguiendo la conocida regla de Naegele para calcular la fecha del parto, solamente el $4.6 \%$ de los nacimientos ocurren el día esperado y el $85.1 \%$ dentro de los siguientes 14 días. Stewart (42), por estudios basados en la determinación de la fecha de la ovulación mediante la temperatura basal, comprobó que la duración real del embarazo no sobrepasó los 285 días, a pesar de que la duración aparente llegara a los 349 días.

Podría deducirse de lo anterior que en realidad el embarazo prolongado no existe; pero la evidencia de que algunos niños nacidos de gestaciones cronológicamente prolongadas muestren al nacer signos de haber padecido in útero un deficiente aporte de materiales nutritivos para su normal desarrollo, obliga a aceptarlo como una entidad clínica y patológica definida. Casi todos los autores consideran que el embarazo se ha prolongado cuando su duración sobrepasa las 42 semanas - o los 294 días- a partir de la iniciación de la última regla; en esta forma quedarían incluídos aquellos casos de probable ovulación tardía.

Debido a que en la actualidad se desconocen con exactitud los principios que rigen la terminación del embarazo, la etiología de la gestación prolongada aún se ignora. Lo único que se sabe con certeza es que el parto no se inicia en la fecha esperada por ausencia de contracciones uterinas, es decir, por una inercia uterina primitiva. Es probable que sea la consecuencia de una deficiente producción de oxitocina (46), o de un exceso de sustancias antiocitócicas (22), o por falta de respuesta contráctil del miometrio $(33,36)$. Sin

\footnotetext{
* Profesor Asistente del Departamento de Obstetricia y Ginecología (Universidad Nacional).
} 
embargo, persisten conceptos como el del predominio sostenido de la progesterona sobre los estrógenos al final del embarazo $(15,31)$. De ser así, esto nos estaría señalando que la placenta - a lo menos en el aspecto hormonal_ funcionalmente se halla en plena actividad, lo cual se opone al concepto de senilidad placentaria con que suele identificarse el embarazo prolongado. El fenómeno curioso de la "implantación diferida", observado por Eckstein y cols. (13) en algunas especies animales inferiores, ha sido sugerido como causa etiológica en la especie humana, aunque no haya sido demostrado en ella. $\mathrm{El}$ hecho de permanecer inactivo el huevo durante varias semanas antes de implantarse, daría como resultado una duración aparente del embarazo y el producto no podría considerarse biológicamente hipermaduro, como tampoco senil la placenta.

Decíamos que lo que le otorga carta de naturaleza a la entidad es el efecto deletéreo que algunas veces ocasiona al feto. Al nacer el niño puede apreciarse delgado, con facies alerta, mostrando evidencia de haber perdido peso; la piel es seca, agrietada, y el cabello abundante y las uñas largas. Cuando la anoxia ha sido severa el líquido amniótico, las membranas ovulares, el cordón y la piel se hallan teñidos de meconio. Según DiGiacomo y Danek (12) tal cosa sucede en el $31 \%$ de los casos cuando el embarazo ha durado más de 295 días, y según Runge (37) en el $25 \%$ después de los 301 días. Ocasionalmente pueden observarse cambios similares en el recién nacido de término y aún prematuro $(21,29)$.

Estas manifestaciones patológicas, descritas desde tiempo atrás por $\mathrm{Ba}$ llantyn primero y luego por Runge, fueron denominadas por Clifford "síndrome de disfunción placentaria"
(11) y por Sjostedt "síndrome de dismadurez" (41). Como no toda prolongación del embarazo se acompaña de este síndrome, Vermelin y cols. (45) hablan de embarazos prolongados "patológicos" y "fisiológicos" (mejor fuera, "no patológicos"). El que unos embarazos prolongados sean patológicos $y$ otros no, es un aspecto más del problema, todavía no dilucidado. Se presume que la prolongación de la gestación repercute desfavorablemente sobre el feto a través de la placenta al ejercer de manera deficiente sus funciones. Las alteraciones histopatológicas de ese órgano, sin ser específicas, muestran según Hertig (16), envejecimiento del trofoblasto y cambios vasculares. $\mathrm{Pa}$ ra Lepage y Schramm (23) no hay ningún detalle definido que permita, al examen de una placenta dada, afirmar que ella es el testimonio de un embarazo prolongado. Dicen Riviere y Dubecq (35) que la esclerosis de las vellosidades, el espesor desigual del revestimiento sincicial o el aumento de la sustancia fibrinoide, no tienen relación con las condiciones del recién nacido, pero que, en cambio, las lesiones vasculares se observan con más frecuencia cuando el nino presenta manifestaciones de postmadurez. Morin y cols. (26) afirman que en las membranas feto-maternas, especialmente en la caduca, hay una disminución de la cantidad de glucógeno cuando el parto ha sido de término, pero que en los prematuros 0 en aquellos postdatados ese glucógeno está dos veces aumentado, lo cual les induce a pensar que en los embarazos prolongados la caduca probablemente no ha llegado a su madurez, lo que explicaría el retraso del parto.

La muerte fetal antes de iniciarse el trabajo de parto, y achacable a la prolongación del embarazo, es poco 
frecuente; al decir de algunos, no tiene mayor diferencia con la observada en las gestaciones de término $(14,29)$. Indudablemente, el período crítico para el feto se identifica con el parto mismo. Para Lindell (24) la mortalidad perinatal aumenta con cada semana que se prolongue el embarazo; para Browne (6) es, en relación con la observada al término, doble a las 43 semanas, triple a las 44 semanas y más del quíntuple a las 45. Hay, además, otros factores que asociados al embarazo prolongado agravan el pronóstico fetal, tales como la toxemia o la primiañosidad.

Dos interrogantes se nos plantean cuando estamos frente a un embarazo que continúa más allá de la fecha prevista para el parto. ¿Se trata realmente de un embarazo prolongado? $\mathrm{Si}$ en verdad lo es, ¿la función placentaria es deficiente?

A pesar del interés puesto para descubrir anteparto si un embarazo es cronológicamente prolongado, hasta el momento no contamos con recursos que nos permitan aclarar la duda. Para calcular la edad del embarazo, en la práctica hemos de basarnos siempre en el dato de la última menstruación ya que -como dice Nesbitt (27) - persiste como el principal denominador, habida consideración de las limitaciones obvias para el diagnóstico. Desde el punto de vista clínico los datos que podemos recoger, aisladamente 0 en conjunto, como la disminución de peso de la embarazada, la regresión de la altura uterina y del perímetro abdominal, son elementos diagnósticos aleatorios. Muchos obstetras se confían en la madurez del cuello uterino para juzgar si el embarazo está de término o no. Al respecto, Browne (7) opina que la madurez cervical es simple evidencia de que el útero está listo para actuar, y mientras en la mayoría de los casos esto coincide con la madurez fetal, no siempre es así; un cuello inmaduro -afirma él- no indica que el feto no esté maduro. Si aceptamos, como lo sostienen Caldeyro y Alvarez (8), que la madurez del cuello es debida a un aumento progresivo de la actividad uterina durante la última etapa del embarazo, debemos convenir que si esa actividad creciente no se presenta, el cuello no sufrirá el proceso normal de maduración. Como vimos ya, hay quienes creen que el embarazo prolongado es un problema de inercia uterina; de ser así, el cuello uterino seguirá siendo inmaduro a pesar de que el embarazo sea realmente prolongado y su contenido hipermaduro.

Algunos, al fracasar la inducción en una paciente con sospecha de embarazo prolongado, concluyen por eso que la gestación no era de término, olvidando que el llamado "test de la inducción" no es un procedimiento para juzgar la edad cronológica del embarazo. Hay tanta variación en la respuesta individual a la oxitocina en los embarazos prematuros, de término o postmaduros, que al decir de Pinedo (30) priva de todo valor a la prueba de la ocitocina.

El estudio radiográfico del feto para buscar ciertos núcleos de osificación es documento de dudosa práctica. La presencia del núcleo de Beclard, correspondiente a la extremidad distal del fémur, y que ha servido para valorar la madurez del feto, fue observado por Schreiber y cols. (40) en el $51 \%$ de los prematuros. Sin embargo, para Schaffer (39) la asociación del núcleo de Beclard con el proximal de la tibia estaría a favor de un feto de término o postmaduro. Además, la calcificación de la placenta —considerada por Blair Hartley como un heraldo de la muer- 
te fetal- es, según Tindall y Scott (44), un proceso natural y fisiológi$\mathrm{co}$, sin ningún valor para el pronóstico fetal o el diagnóstico de la entidad.

El estudio de la citología vaginal y del sedimento urinario es, al parecer, de utilidad práctica para determinar la madurez del embarazo. Se sabe que en las últimas semanas la caída de los niveles de progesterona y el predominio de los estrógenos se reflejan en el epitelio urogenital $y$, por lo tanto, pueden identificarse por los cambios citológicos. Alvarez Bravo y cols. (3) afirman que es posible predecir la proximidad del parto en casi el $100 \%$ de los casos cuando hay un aumento del índice acidófilo y cariopicnótico, presencia de desvitalización celular, desaparición de los grupos de células aglutinadas y existencia de células intermediarias acidófilas. Para Sammour (38) el pronóstico fue acertado en el $83 \%$ de los casos y para Barnes y Zuspan (5) en el 73\%. Este método, aceptable para juzgar la proximidad del parto, no permite, según manifiestan Castaño (10) y Jorio (20), predeterminar las condiciones fetales. Por el contrario, hay quienes dicen que en las gestaciones realmente prolongadas es posible hallar en el extendido vaginal un cuadro regresivo caracterizado por la presencia de células parabasales, semejante al que se observa en el puerperio precoz, lo que permitiría por sí solo hacer el diagnóstico y el pronóstico $(9,25,47)$.

No se ha publicado hasta el momento ningún estudio definido sobre el empleo de la biopsia placentaria en el embarazo prolongado. Solamente Alvarez y cols. (4) hacen referencia a algunos casos con diagnóstico dudoso en que la biopsia descartó alteraciones placentarias. Es claro, sin embargo, que este procedimiento tiene el inconveniente de no poderse utilizar en todas las ocasiones ya que el éxito de la biopsia está supeditado al sitio de inserción placentaria.

Herrera y cols. (17), suponiendo que en el embarazo prolongado la alteración del tejido placentario podría manifestarse en los niveles de las transaminasas séricas, emprendieron la investigación de la glutámica oxalacética y observaron que tanto en los embarazos normales como en los prolongados en que se halló meconio al nacimiento estuvo elevada, aunque no encontraron una relación constante entre las cifras altas y el aspecto de postmadurez del niño.

Uno de los recursos que al parecer permite diagnosticar de manera incontrovertible durante el embarazo aquellos casos de gestaciones prolongadas patológicas, es la amnioscopia. Jantzen (19) afirma que es posible, observando a través del cuello uterino el polo inferior del huevo, determinar la cantidad y el aspecto del lí. quido amniótico. La comprobación de escaso líquido es un signo a favor de una prolongación incipiente; en cambio, la tinción con meconio es un signo de alarma, y según la homogeneidad de ella puede presumirse el tiempo que lleva el feto en hipoxia. Otro dato de valor es la adherencia del polo inferior del huevo al orificio interno del cuello, que de estar presente niega el diagnóstico de embarazo prolongado. Agüero (2) también le asigna valor al procedimiento, que él denomina "histeroscopia".

En relación con la conducta obstétrica que ha de seguirse, las opiniones están divididas. Rathbun (32), por ejemplo, dice que el parto solo debe provocarse cuando el cuello esté maduro, ya que no se justifica guardar temor de que el feto pueda perderse in utero; en contraposición, 
hay quienes inducen el parto sistemáticamente 7 a 10 días después de la fecha prevista $(1,43)$ y otros lo hacen si la prolongación se acompaba de circunstancias especiales como toxemia, primiañosidad, antecedentes de nacidos muertos, etc. $(7,34)$. Finalmente, existen los que aconsejan cive la respuesta al problema no es una indiscriminada y rutinaria inducción, sino la anticipación y el rápido manejo de las complicaciones que surjan durante el curso del parto $(27,31)$.

\section{CONCLUSION}

De lo expuesto en la revisión anterior se saca en claro que el "embarazo prolongado" es una entidad clínica definida, cuya etiología permanece todavía ignorada. Es cierto que por no existir evidencia del momento en que el embarazo se inicia, nos es difícil -o mejor, imposible-, juzgar cuándo es realmente prolongado. A pesar de lo veleidoso que sea el dato de la última menstruación, tenemos que continuar tomándolo como punto de partida para calcular la edad del embarazo y para determinar la fecha aproximada del parto; así, solo cuando han transcurrido 42 semanas ha de presumirse que la gestación comienza a prolongarse.

De los recién nacidos de embarazos cronológicamente prolongados, apenas un 20 a $30 \%$ exhiben manifestaciones de postmadurez, algunos de ellos con signos claros de daño anóxico. Por lo tanto, es el recién nacido el que obliga a considerar patológico o no el embarazo prolongado.

En la práctica no contamos aún con recursos que, frente a un caso dado, nos permitan diagnosticar en el período antenatal la prolongación del embarazo. Una adecuada interpretación de los hallazgos citológi- cos en un extendido vaginal podrá informarnos si la madurez del embarazo ha llegado a su término $y$, ocasionalmente, descubrir la presencia de elementos celulares regresivos. La amnioscopia, que está todavía en la etapa de investigación, pondría de presente las condiciones fetales $y$, por deducción, el grado de disfunción placentaria.

Como corolario, parece que la conducta aconsejable ante un embarazo que haya transpuesto las 42 semanas sería proceder a la inducción del trabajo, teniendo presente que el momento de mayor peligro para la vida del feto es, precisamente, el que corresponde al parto.

\section{BIBLIOGRAFIA}

1 Aguero, O. y ROMERO, A. Rev. Obst. Gin. Venezuela. 19:307, 1959.

2 AGUero, O., AURE, M. y LOPEZ, R. Am. J. Obst. \& Gynec. 94:925, 1966.

3 ALVAREZ, A., GONZALEZ, M., GUTIERREZ, E. y DOSAL, M. Ginec. Obstet. Mex. 12:73, 1957.

4 ALVAREZ, H., BEJAR, R., ALADJEM, S. y otros. "La Placenta Humana". Mem. Cuarto Cong. Uruguayo de Ginecot., Tomo I, pág. $190,1964$.

5 BARNES, A. C. Y ZUSPAN, F. P. Am. J. Obst. \& Gynec. $71: 1080,1956$.

6 BROWNE, J. C. Brit. Med. J. 2:1080, 1962.

7 BROWNE, J. C. J.A.M.A. 186:1047, 1963.

8 CALDEYRO, R. y ALVAREZ, H. "Juicio crítico de la inducción y conducción del parto". Mem. Tercer Cong. Obst. Gin., México, Tomo I, pág. 140, 1958.

9 CARRERAS, L. y MILLANI, L. Quad. clin. ostet. e ginec. $12: 227,1957$.

10 CAStaño, A. A. Acta Gynaec. et Obstet. Hisp. Lus. 3:155, 1963.

11 CLIFFORD, S. H. J. Pediat. $44: 1,1954$.

12 DIGIACOMO, B. E. y G. DANEK, G. Min. Ped. $10: 834,1958$. 
13 ECKSTEIN, P., SHELESKYAK, M. C. Y AMOROSO, E. "Implantatio of ova". Mem. Soc. Endocr. No 6, Cambridge Univ. Press, 1959.

14 EVANS, T. N., KOEFF, S. T. Y MORLEY, G. N. Am. J. Obst. \& Gynec. 85:701, 1963.

15 FIRPO, J. R. Excerpta Medica, Obst. and Gynec. 2:376, 1949.

16 HERTIG, A. T. "Pathological aspects". en "The Placenta and Fetal Membranes". C. Ville, pág. 120, 1960.

17 HERRERA, M., DE LA FUENTE, S., UGARTE, G. y LORCA, F. Rev. Chilena de Obst. y Gin. $27: 141,1962$.

18 HIGGINS, L. G. J. Obst. \& Gynaec. Brit. Emp. 63:567, 1956.

19 JANTZEN, K. Landarzt. 16:665, 1965.

20 JORIO, A. Rass. Int. Cl. Terap. 41:1170, 1961.

21 KUNSTADTER, R. H. y SCHNITZ, S. E. J. A.M.A. $161: 1551,1956$.

22 LAMBRINOPOULUS, T. C. Obst. \& Gynec. $23: 780,1964$.

23 LEPAGE, F. y SCRAMM, B. Bull. Fed. Soc. Gynec. et Obst. 9:415, 1957.

24 LINDELL, A. Acta obst. et gynec. scandinav. $35: 136,1956$.

25 MATEU-ARAGONES, J. M. Rev. Española Obst. Gin. 125:373, 1962.

26 MORIN, P., HANOU, F., COQUOIN, M. Y RAUX, J. M. Gynec. et Obstet. 57:90, 1958.

27 NESBITT, R. E. L. Obst. \& Gyn. Surv. 10 $311,1955$.

28 NESBITT, R. E. L. J.A.M.A. 165:1656, 1957.

29 PERLIN, I. A. Am. J. Obst. \& Gynec. 80 1,1960 .
30 PINEDO, G. Rev. Obst. Gin. Venezuela. 22: $687,1962$.

31 RAMIREZ, C. Bol. Soc. Chilena Obst. y Ginec. $18: 101,1953$.

32 RATHBUN, L. S. Am. J. Obst. \& Gynec. $46: 278,1943$.

33 REENKOLA, M. Acta Obst. et gynec. scandinav. 28, Suppl, 3, 1948.

34 REIS, R. A. Post grad. Med. $35: 333,1964$

35 RIVIERE, M. y DUBECQ, J. P. Bull. Fed. Soc. Gynec. et Obst. 9:417, 1957.

36 RUNGE, H. Deutsche. Med. Woch. 541, 1939 .

37 RUNGE, H. Y BACH, H. G. Deutsche. Med. Woch. 83:1811, 1958.

38 SAMMOUR, M. B. Obst. \& Gynec. 24:682, 1964.

39 SCHAFFER, B. Arch. Gin. y Obst. Uruguay. $16: 95,1958$

40 SCHREIBER, M. H., MENACHCF, L., GUNN, W. G. Y BIEHUSEN, F. C. Am. J. Obst. \& Gynec. 83: 1249, 1962.

41 SJOSTEDT, S., ENGLESON, G. y ROUTH, G. Arch. Dis. Child. $33: 168,1958$.

42 STEWART, H. L. J.A.M.A. 148:1079, 1952.

43 THEOBALD, G. W. Lancet. 1:59, 1959.

44 TINDALL, V. R. y SCOTT, J. S. J. Obst. \& Gynaec. Brit. Emp. 72:356, 1965.

45 VERMELIN, H., RICHON, J. Y RIBON, C. M. Bull. Fed. Soc. Gyn. et Obst. 9:413, 1957.

46 VLYSSIDES, Z. y ZERVOUDAKIS, A. Bull. Fed. Soc. Gyn. et Obst. 9:401, 1957.

47 WALTER, P. y LOPEZ, E. Bull. Fed. Soc. Gyn. et Obst. 9:443, 1957. 